\title{
SEMIGROUPS SATISFYING MINIMAL CONDITIONS
}

\author{
by W. D. MUNN
}

(Received 28 June, 1957)

In his fundamental paper, "On the structure of semigroups" [6], J. A. Green has examined certain important minimal conditions which may be satisfied by a semigroup $S$. We say that $S$ satisfies the minimal condition on principal left ideals if every set of principal left ideals of $S$ contains a minimal member with respect to inclusion : this condition is denoted by $\mathscr{H}_{l}$. The corresponding conditions on principal right ideals and principal two-sided ideals are denoted by $\mathscr{H}_{\mathrm{r}}$ and $\mathscr{H}_{f}$ respectively. The purpose of the present paper is to give some further results concerning these three conditions. Extensive use is made of the work of A. H. Clifford ([3] and [4]) on minimal ideals.

The discussion falls into three sections. The first of these contains a preliminary account of the conditions $\mathscr{H}_{l}, \mathscr{H}_{\tau}$ and $\mathscr{H}_{f}$, in the course of which it is pointed out that they are, in general, independent. It is shown, however, that in a semisimple semigroup $\mathscr{M}_{l}$ implies $\mathscr{M}_{f}$ (Theorem 1.2).

In the second section we introduce the concept of a completely semisimple semigroup. This is a semigroup whose principal factors are all completely simple. Such a semigroup is necessarily regular, but the converse is not true. We also define two further conditions, $\mathscr{M}_{l}^{*}$ and $\mathscr{H}_{r}^{*}$, which are similar to, but weaker than $\mathscr{M}_{l}$ and $\mathscr{M}_{r}$ respectively. These are used in the formulation of alternative criteria for complete semisimplicity (Theorems 2.3 and 2.5); we deduce that a semisimple semigroup satisfying $\mathscr{M}_{l}$ and $\mathscr{M}_{r}$ is completely semisimple, and that a regular semigroup satisfying $\mathscr{H}_{l}$ is completely semisimple. Semigroups which admit relative inverses (Clifford, [2]) form an important class of completely semisimple semigroups. With the aid of a result due to $R$. Croisot [5], these are given a new characterisation in Theorem 2.7: it follows from this that if $S$ is a semigroup satisfying $\mathscr{M}_{l}$ and such that every element $a \in S$ belongs to the set $a^{2} S$, then $S$ admits relative inverses. Finally, it is shown that in a completely semisimple semigroup the conditions $\mathscr{M}_{l}, \mathscr{M}_{r}$ and $\mathscr{M}_{f}$ are all equivalent (Theorem 2.9).

The last section deals with the topic of radicals in a semigroup, again with emphasis on results dependent on minimal properties. First we define the "upper radical " of a semigroup $S$ to be the intersection of all the ideals $M$ such that $S / M$ is semisimple; if this is non-empty it may be described as the unique smallest ideal $N^{\prime}$ such that $S / N^{\prime}$ is semisimple (Theorem 3.1). It follows from the results of the previous section that if every element of $S$ has finite order, or if $S$ satisfies $\mathscr{M}_{l}$ and $\mathscr{M}_{r}$, then $S / N^{\prime}$ is completely semisimple. By contrast, corresponding to any ideal $M$ we define the "M-radical " $N_{M}$ to be the union of all the $M$-potent ideals of $S$. This radical is of a type previously studied by Schwarz [10] and by Clifford [4]. If $N_{M}$ is $M$-potent, then $S / N_{M}$ is a semigroup without nilpotent ideals. We show that if such a semigroup satisfies any one of the conditions $\mathscr{M}_{l}, \mathscr{M}_{r}, \mathscr{M}_{f}$, then every non-zero ideal contains a non-zero simple ideal (Theorem 3.4). This result yields two corollaries which give conditions for the $M$-potence of a semigroup $S$, where $M$ is an ideal of $S$.

1. The conditions $\mathscr{M}_{l}, \mathscr{M}_{r}$ and $\mathscr{H}_{f}$. By a semigroup we shall mean a set which is closed under a single associative binary operation : this will be denoted by juxtaposition in the usual way. 
We recall some definitions given by Green [6]. The elements $a$ and $b$ of a semigroup $S$ are said to be I-equivalent if $a$ and $b$ generate the same left ideal of $S$; that is, if $S a \cup a=S b \cup b$. In the same way, two elements are r-equivalent if they generate the same right ideal of $S$, and \{-equivalent if they generate the same two-sided ideal of $S$. For any $a \in S$ we shall denote by $L_{a}, R_{a}$ and $F_{a}$ the I-, $\mathfrak{r}$ - and f-classes respectively to which $a$ belongs. Evidently $L_{a} \subseteq F_{a}$ and $R_{a} \subseteq F_{a}$.

Let $I$ be a principal (that is, simply generated) ideal of $S$, and let $J$ be its set of nongenerators : then $I-J$, the set of elements in $I$ but not in $J$, is an f-class of $S$, say $F$. It can easily be shown that if $J$ is non-empty it is an ideal of $S$, and we call the Rees quotient semigroup $I / J$ the principal factor of $S$ corresponding to $F$. This is either simple, or else its square is zero. If it is simple, then we say that $F$ is a simple $f$-class ; this is the case if and only if every element $a \in F$ belongs to the set $F a F$ ([6], Theorem 5). If all the f-classes of $S$ are simple, then $S$ is said to be semisimple.

It may happen that the intersection $K$ of all the ideals of $S$ is non-empty; if this is so, we call $K$ the kernel of $S$ (Clifford, [3]). It is a principal ideal, and when $I=K$ in the above discussion, the set $J$ is empty. We shall make the convention, however, that $I / J=I$ in this case, and include it amongst the principal factors. The kernel is a simple f-class of $S$.

The I-classes of $S$ may be given a natural partial ordering as follows. Let $L$ and $L^{\prime}$ be I-classes ; then we write $L \leqslant L^{\prime}$ if and only if the left ideal of $S$ generated by elements of $L$ is contained in the left ideal generated by elements of $L^{\prime}$. If every set of I-classes of $S$ contains a minimal member with respect to this partial ordering, then we shall say that $S$ satisfies the condition $\mathscr{H}_{l}$. This means that every set of principal left ideals contains a minimal member with respect to inclusion, or, equivalently, that every strictly descending chain of principal left ideals of $S$ breaks off after a finite number of terms. As remarked by Green, $\mathscr{H}_{l}$ is weaker than the minimal condition on all left ideals of $S$, principal and otherwise.

Partial orderings of the $r$ - and f-classes of $S$ (which we shall also denote by the symbol $\leqslant)$, and the corresponding minimal conditions $\mathscr{H}_{r}$ and $\mathscr{H}_{f}$, are defined in a similar manner.

THEOREM 1.1. Let $S$ be a semigroup satisfying any one of the conditions $\mathscr{M}_{l}, \mathscr{H}_{n}, \mathscr{M}_{g}$. Then $S$ has a kernel.

Proof. Suppose first that $S$ satisfies $\mathscr{M}_{f}$. From the set of all f-classes of $S$ select a minimal member $K$. Then $K$ is a minimal ideal of $S$, and so it is the kernel of $S([3], \S 1)$.

Now suppose instead that $S$ satisfies $\mathscr{M}_{l}\left[\mathscr{M}_{+}\right]$. Then the set of all 1 -[r-] classes of $S$ contains a minimal member, and this is a minimal left [right] ideal of $S$. Hence $S$ has a kernel, namely the union of all the minimal left [right] ideals ([3], Theorem 2.1).

In particular, the kernel may consist of a single element; this element is then the zero of $S$.

The conditions $\mathscr{M}_{l}, \mathscr{H}_{r}$ and $\mathscr{M}_{f}$ are, in general, quite independent. It is not difficult to construct semigroups which satisfy $\mathscr{M}_{f}$ but which fail to satisfy $\mathscr{H}_{l}$ and $\mathscr{H}_{r}$. Less immediate, perhaps, is the fact that a semigroup may satisfy $\mathscr{M}_{l}$ without satisfying $\mathscr{H}_{r}$ and $\mathscr{M}_{f}$.

Example. Let $S$ be the set consisting of an element 0 , together with the set of all ordered pairs $(i, j)$, where $i$ and $j$ are positive integers such that $i<j$. Multiplication in $S$ is defined by the rules

$$
(i, j)(r, s)=\left\{\begin{array}{cl}
(i, s) & \text { if } j=r \\
0 & \text { if } j \neq r
\end{array} ; \quad 0 x=0=x 0 \text { for all } x \in S\right.
$$


This is associative, and so $S$ is a semigroup. The left ideal generated by $(i, j)$ is

$$
\{0 ;(r, j), 1 \leqslant r \leqslant i\} ;
$$

since this set is finite, $S$ satisfies $\mathscr{M}_{l}$. Similarly, the right ideal $R_{i j}$ generated by $(i, j)$ is $\{0 ;(i, s), s \geqslant j\}$, and the two-sided ideal $I_{i j}$ generated by $(i, j)$ is $\{0 ;(r, s), 1 \leqslant r \leqslant i, s \geqslant j\}$. But the strictly descending sequences $R_{i j} \supset R_{i, j+1} \supset R_{i, j+2} \supset \ldots$ and $I_{i j} \supset I_{i, j+1} \supset I_{i, j+2} \supset \ldots$ can both be continued indefinitely : hence $S$ satisfies neither $\mathscr{M}_{r}$ nor $\mathscr{M}_{f}$.

We note, in addition, that the semigroup first discussed by Baer and Levi ([1], p. 7) provides an example which satisfies both $\mathscr{M}_{l}$ and $\mathscr{M}_{f}$ (since it consists of a single [-class), but which does not satisfy $\mathscr{M}_{r}$. On the other hand, Green has shown that $\mathscr{M}_{l}$ and $\mathscr{M}_{r}$ together imply $\mathscr{H}_{f}([6]$, Theorem 4).

It will now be shown that the situation illustrated by the example given above cannot occur in a semisimple semigroup.

ThEOREm 1.2. Let $S$ be a semisimple semigroup satisfying $\mathscr{M}_{l}$. Then $S$ satisfies $\mathscr{M}_{f}$.

Proof. Let $\mathcal{f}$ be any set of $f$-classes of $S$, and let $\mathbb{L}$ be the set of all I-classes of $S$ which are contained in members of $\mathcal{f}$. Then since $S$ satisfies $\mathscr{M}_{l}, \mathfrak{L}$ contains a minimal member. Let this be $L_{a}$, for some $a \in S$. We shall show that $F_{a}$ is minimal in $f$. Suppose this is false; then there exists an element $b \in S$ such that $F_{b} \in f$ and $F_{b}<F_{a}$. From the latter we see that $b \in S a \cup a S \cup S a S$. Also since $S$ is semisimple, $F_{b}$ is simple and so $b \in F_{b} b F_{b}$ ([6], Theorem 5). Hence $b \in F_{b}(S a \cup a S \cup S a S) F_{b}$, from which it follows easily that $b \in F_{b} a F_{b}$. Let $u$ and $v$ be elements of $F_{b}$ such that $b=u a v$. Then $F_{u a}=F_{b} \in \mathcal{F}$, and so $L_{u a} \in \mathfrak{S}$. But $L_{u a} \leqslant L_{a}$; hence since $F_{b}<F_{a}$ we have $L_{u a}<L_{a}$, which contradicts the minimality of $L_{a}$ in 5. Thus $F_{a}$ is minimal in $\mathcal{f}$; that is, $S$ satisfies $\mathscr{M}_{f}$.

2. Completely semisimple semigroups. Let $S$ be a semigroup and $T$ a subset of $S$. An idempotent $e \in T$ will be said to be primitive in $T$ if the only idempotent in $T$ satisfying the equations $e x=x=x e$ is $e$ itself. We shall then say that an f-class $F$ of $S$ is completely simple if it contains an idempotent which is primitive in $F$. This holds if and only if the corresponding principal factor is a completely simple semigroup in the sense of Rees ([8] and [9]); the structure of such semigroups has been fully determined ([8], Theorem 2.93).

Definition. A completely semisimple semigroup is a semigroup in which each f-class is completely simple.

Evidently a finite semisimple semigroup is completely semisimple. More generally, a semisimple semigroup in which every element has finite order is completely semisimple; for each principal factor is then a simple semigroup in which every element has finite order, and Rees has shown that such semigroups are completely simple ([8], Lemma 2.4). As was noted by Green, every element of a semigroup $S$ has finite order if and only if $S$ satisfies the minimal condition on principal subsemigroups. Another important class of completely semisimple semigroups comprises those semigroups which admit relative inverses : these are discussed in Theorem 2.7 below.

Green defines a semigroup $S$ to be regular if every element $a \in S$ is such that $a \in a S a$. It is a consequence of Rees's structure theorem for completely simple semigroups that a completely semisimple semigroup is regular. Furthermore, a regular semigroup is semisimple. However, it is easy to find examples which show that neither of the reverse implications is true.

We shall make frequent use of the following elementary property of semigroups : 
Lemma 2.1. Let $S$ be a semigroup and $M$ an ideal of $S$. Then the $I_{-}\left[\mathfrak{r}_{-}, f-\right]$ classes of $S$ contained in the set $S-M$ are just the non-zero $I_{-}[\mathrm{r}-, \mathrm{f}-]$ classes of $S / M$.

The proof is trivial. Suppose that $a$ and $b$ are distinct elements of $S-M$. Then the criteria for their I-equivalence qua elements of $S$ and qua elements of $S / M$ are the same, namely that there exist elements $x$ and $y$ in $S-M$ such that $a=x b$ and $b=y a$. Similar arguments apply to $r$. and f-equivalence.

In particular, this result shows that if $S$ satisfies $\mathscr{M}_{l}, \mathscr{M}_{r}$ or $\mathscr{M}_{f}$, then so also does $S / M$.

It is convenient at this stage to introduce two further minimal conditions, $\mathscr{H}_{l}^{*}$ and $\mathscr{M}_{r}^{*}$. A semigroup $S$ will be said to satisfy $\mathscr{H}_{l}^{*}\left[\mathscr{H}_{r}^{*}\right]$ if the set of all I-[r-] classes of $S$ contained in any $f$-class of $S$ contains a minimal member. Clearly $\mathscr{H}_{l}$ implies $\mathscr{H}_{l}^{*}$ and $\mathscr{H}_{r}$ implies $\mathscr{H}_{r}^{*}$.

In the proof of the next lemma, and subsequently, we follow Clifford's convention as regards the use of the term "minimal left [right, two-sided] ideal". Thus in a semigroup $S$ with zero, a minimal left ideal is a non-zero left ideal of $S$ properly containing no left ideal of $S$ other than the zero ideal.

LEMma 2.2. Let $S$ be a semigroup satisfying $\mathscr{H}_{l}^{*}$, and let $F$ be any f-class of $S$. Then every 1 -class of $S$ contained in $F$ is minimal in the set of all I-classes contained in $F$.

Proof. Let $I$ be the ideal generated by $F$, and let $J=I-F$. Then $J$ is an ideal $\dagger$ of $S$, and $I / J$ is a minimal ideal of $S / J$. Since $S$ satisfies $\mathscr{H}_{l}^{*}$, so also does $S / J$ by Lemma 2.1, and thus $I / J$ contains a minimal left ideal of $S / J$. Hence $I / J$ is a union of minimal left ideals of $S / J$ ([4], Theorem 2.1), and so every principal left ideal of $S / J$ generated by a nonzero element of $I / J$ is minimal in $S / J$. Thus every I-class of $S / J$ contained in $F$ is minimal in the set of all l-classes contained in $F$; but by Lemma 2.1 these are also l-classes of $S$, and hence we have the required result.

This lemma is similar to part (ii) of Green's Theorem 8, but is more general.

We now go on to obtain two alternative characterisations of complete semisimplicity involving minimal conditions. The first of these depends directly on results due to Clifford [4].

THeOREM 2.3. A semigroup $S$ is completely semisimple if and only if it is semisimple and satisfies the conditions $\mathscr{M}_{l}^{*}$ and $\mathscr{H}_{r}^{*}$.

Proof. Suppose that $S$ is completely semisimple. Let $F$ be any f-class of $S$, let $I$ be the ideal generated by $F$, and let $J=I-F$. Then $I / J$ is completely simple. Hence $I / J$ contains a minimal left ideal and a minimal right ideal of itself ([4], Theorem 3.2). Now since $I / J$ is simple, a minimal left ideal of $I / J$ is also a minimal left ideal of $S / J$ ([4], Theorem 2.2 ; also [4], reference 3, p. 844), and so its non-zero elements constitute an I-class of $S / J$. Thus the set of all I-classes of $S / J$ contained in $F$ contains a minimal member, and hence by Lemma $2.1 S$ satisfies $\mathscr{M}_{l}^{*}$. Similarly, $S$ satisfies $\mathscr{M}_{r}^{*}$.

Conversely, suppose that $S$ is semisimple and satisfies $\mathscr{M}_{l}^{*}$ and $\mathscr{M}_{r}^{*}$. Let $F, I$ and $J$ be defined as above. Then $S / J$ satisfies $\mathscr{H}_{l}^{*}$ and $\mathscr{M}_{r}^{*}$ by Lemma 2.1. Hence $I / J$ is a minimal simple ideal of $S / J$ containing a minimal left ideal and a minimal right ideal of $S / J$, and so $I / J$ is completely simple ([4], Theorem 3.1). Thus $S$ is completely semisimple.

CoRollary 2.4. A semisimple semigroup satisfying $\mathscr{H}_{l}$ and $\mathscr{M}_{r}$ is completely semisimple.

Green ([6], p. 17I) has shown that such a semigroup is regular.

The next result gives conditions for complete semisimplicity which are intermediate between those of the definition and those provided by Theorem 2.3.

† Here, as in the proof of Theorem 2.3, if $F$ is the kernel of $S$ (that is, if $J$ is empty), then we make use of results from [3] in place of the corresponding results from [4]. 
Theorem 2.5. A semigroup is completely semisimple if and only if it satisfies $\mathscr{M}_{l}^{*}$ and has the property that every $f$-class contains an idempotent.

Proof. Let $S$ be a completely semisimple semigroup. Then by Theorem 2.3 it satisfies $\mathscr{H}_{l}^{*}$, and from the definition every f-class contains an idempotent.

Conversely, let $S$ be a semigroup satisfying $\mathscr{M}_{l}^{*}$, and such that every f-class contains an idempotent. Let $e$ be any idempotent of $S$, and let $f$ be an idempotent in $F_{e}$ with the property that $e f=f=f e$. From the relation $f e=f$ we see that $L_{f} \leqslant L_{e}$. Now since $S$ satisfies $\mathscr{H}_{l}^{*}$, it follows from Lemma 2.2 that $L_{e}$ is minimal in the set of all I-classes contained in $F_{e}$. Thus $L_{f}=L_{e}$. Hence in particular, there is an element $a \in S$ such that $e=a f$, and so

$$
e f=a f^{2}=a f=e \text {. }
$$

But $e f=f$; hence $e=f$. Thus $e$ is primitive in $F_{e}$, and so $S$ is completely semisimple.

A special case of this theorem has previously been obtained by Schwarz [11], who showed that a simple semigroup without zero is completely simple if and only if it contains a minimal left ideal and an idempotent.

\section{CoROLLARY 2.6. A regular semigroup satisfying $\mathscr{M}_{l}$ is completely semisimple.}

We need only note that each $f$-class of a regular semigroup contains an idempotent ([6], Theorem 6).

This should be compared with Corollary 2.4. By replacing semisimplicity by the stronger requirement of regularity we have been able to remove one of the minimal conditions.

A semigroup $S$ is said to admit relative inverses if, corresponding to any element $a \in S$, there are elements $e$ and $a^{\prime}$ in $S$ such that $e a=a=a e$ and $a^{\prime} a=e=a a^{\prime}$. Clifford characterises such a semigroup first as a union of disjoint groups ([2], Theorem 1), and then, effectively, as a completely semisimple semigroup in which each $f$-class is a subsemigroup ([2], Theorem 2).

Another characterisation has been obtained by Croisot [5] as a result of his generalisation of the concept of regularity. A semigroup $S$ is said to satisfy the condition $(m, n)$, where $m$ and $n$ are non-negative integers such that $m+n \geqslant 2$, if any element $a \in S$ belongs to the set $a^{m} S a^{n}$. (The condition ( $\left.m, 0\right)$ is satisfied by $S$ if $a \in a^{m} S$ for all $a \in S$.) Croisot has shown that with respect to logical equivalence the set of all such conditions falls into four classes, from which we may select as representatives the conditions $(1,1),(2,0),(0,2)$ and $(2,1)$. He has proved, in addition, that a semigroup admits relative inverses if and only if it satisfies both $(2,0)$ and $(0,2)$, and that these are together equivalent to the single condition $(2,1)$ ([5], Theorems 1 and 2). We now show that the result still holds if we replace one of the conditions $(2,0)$ and $(0,2)$ by a suitable minimal condition.

Theorem 2.7. A semigroup admits relative inverses if and only if it satisfies the conditions $(2,0)$ and $\mathscr{H}_{l}^{*}$.

Proof. Let $S$ be a semigroup which admits relative inverses, and let $a \in S$. Then there exist elements $e$ and $a^{\prime}$ in $S$ such that $a e=a$ and $a a^{\prime}=e$. Hence $a=a\left(a a^{\prime}\right) \in a^{2} S$; that is, $S$ satisfies $(2,0)$. Further, as remarked above, $S$ is completely semisimple ([2], Theorem 2$)$, and so satisfies $\mathscr{H}_{l}^{*}$ by Theorem 2.3 .

Conversely, suppose that $S$ satisfies $(2,0)$ and $\mathscr{H}_{l}^{*}$. We shall prove that $S$ admits relative inverses by showing that it also satisfies $(0,2)$; the result will then follow from Croisot's Theorem 1. Let $a \in S$; then since $a \in a^{2} S$ we have $F_{a} \leqslant F_{a^{2}}$. But $F_{a^{2}} \leqslant F_{a}$, and so $F_{a^{2}}=F_{a}$. This shows that $L_{a^{2}} \subseteq F_{a}$. Now $L_{a^{2}} \leqslant L_{a} ;$ hence since $S$ satisfies $\mathscr{M}_{l}^{*}$, it follows 
from Lemma 2.2 that $L_{a^{2}}=L_{a}$. Thus either $a=a^{2}$ or $a \in S a^{2}$, and the former case is included in the latter, since $a=a^{2}$ implies that $a=a . a^{2}$. Hence $S$ satisfies $(0,2)$, and this completes the proof.

COROLLARY 2.8. A semigroup satisfying $(2,0)$ and $\mathscr{M}_{1}$ admits relative inverses.

Theorem 2.7 no longer holds if we replace $(2,0)$ by $(0,2)$. This may be illustrated by the semigroup of Baer and Levi [1], which consists of a single I-class, and so satisfies both $(0,2)$ and $\mathscr{M}_{l}^{*}$, but which is not completely simple.

We end the present section with a result linking the conditions $\mathscr{M}_{l}, \mathscr{M}_{\tau}$ and $\mathscr{M}_{f}$ in a completely semisimple semigroup.

THEOREM 2.9. If a completely semisimple semigroup satisfies one of the conditions $\mathscr{H}_{l}$, $\mathscr{H}_{r}, \mathscr{H}_{\rho}$, then it satisfies the others.

Proof. We have already shown in Theorem 1.2 that in a semisimple semigroup $\mathscr{H}_{1}$ implies $\mathscr{M}_{f}$. It will be sufficient to prove that in a completely semisimple semigroup $\mathscr{M}_{f}$ implies $\mathscr{M}_{l}$. The remaining cases follow from the left-right duals of these results.

Accordingly, let $S$ be a completely semisimple semigroup satisfying $\mathscr{M}_{f}$, and let $\mathbb{S}$ be any set of I-classes of $S$. Let $\mathcal{f}$ denote the set of all f-classes which contain members of $\mathfrak{L}$. Then since $S$ satisfies $\mathscr{M}_{f}, f$ contains a minimal member, $F$ say. Now let $L$ be any member of $\mathfrak{L}$ which is contained in $F$. We shall show that $L$ is minimal in $\mathbb{L}$. Suppose it is not; then there exists $L^{\prime} \in \mathbb{E}$ such that $L^{\prime}<L$. By Theorem 2.3, $S$ satisfies $\mathscr{H}_{l}^{*}$, and so $L$ is minimal in the set of all I-classes contained in $F$, by Lemma 2.2. Hence $L^{\prime} \Phi F$. Let $F^{\prime}$ be the f-class containing $L^{\prime}$. Then since $L^{\prime}<L$ we have $F^{\prime}<F$, which contradicts the minimality of $F$ in $f$. Hence $L$ must be minimal in $\mathfrak{S}$, and so $S$ satisfies $\mathscr{H}_{l}$, as required.

3. Radicals of a semigroup. In this final section we discuss two types of radical in a semigroup $S$.

First let $N^{\prime}$ denote the intersection of all the ideals $M$ of $S$ which are such that $S / M$ is semisimple. The set of all such ideals is non-empty, since $S$ itself is a member : however, $N^{\prime}$ may be empty, as will happen when $S$ is a semisimple semigroup with no kernel. We shall call $N^{\prime}$ the upper radical of $S$. It has previously been discussed by the author in the case where $S$ has a principal series ([7], $\S 2)$. If $N^{\prime}$ is non-empty, then it is an ideal of $S$.

Theorem 3.1. Let $N^{\prime}$ be the upper radical of a semigroup $S$. Then $S / N^{\prime}$ is semisimple.

Proof. Let $F$ be any non-zero f-class of $S / N^{\prime}$. Then by Lemma $2.1, F$ is also an $f$-class of $S$. But from the definition of $N^{\prime}$ there is an ideal $M$ such that $F \in S-M$ and $S / M$ is semisimple. Hence, again by Lemma $2.1, F$ is an f-class of $S / M$ and so is simple. Thus $S / N^{\prime}$ is semisimple.

As before, if $N^{\prime}$ is empty we take $S / N^{\prime}$ to mean $S$ itself.

Since a semisimple semigroup in which every element has finite order is completely semisimple, we deduce

CoROLLARY 3.2. If every element of $S$ has finite order, then $S / N^{\prime}$ is completely semisimple. Again, from Lemma 2.1 and Corollary 2.4 we have

Corollary 3.3. If $S$ satisfies $\mathscr{M}_{l}$ and $\mathscr{M}_{r}$, then $S / N^{\prime}$ is completely semisimple.

From Theorem 3.1 we see that if $N^{\prime}$ is non-empty it may be described as the unique smallest ideal of $S$ whose quotient semigroup is semisimple. In passing, we note that similar radicals can be defined by replacing the property of semisimplicity in the definition of $N^{\prime}$ by regularity or complete semisimplicity. The appropriately modified form of Theorem 3.1 will 
hold, in fact, if we talke for our definition any property of $S$ which is an intrinsic property of the f-classes-that is, which holds for $S$ if and only if it holds for each principal factor.

We now go on to discuss a second type of radical in $S$. Let $M$ be any ideal of $S$. Then if $A$ is a subset of $S$ such that $A^{n} \subseteq M$ for some positive integer $n, A$ is said to be $M$-potent. In particular, if $S$ has a zero 0 and if $M=(0)$, then we say that $A$ is nilpotent. We define $N_{M}$ to be the union of all the two-sided $M$-potent ideals of $S$, and call it the $M$-radical of $S$; it follows as in [4], Lemma 5.2, that $N_{M}$ contains all the $M$-potent left and right ideals of $S$. In general, $N_{M}$ is not itself $M$-potent ; however, a sufficient condition for this to be the case is that $S$ satisfies the maximum condition on two-sided ideals.

The $M$-radical and the upper radical are related by the inclusion $N_{M} \subseteq N^{\prime} \cup M$. For suppose that $A$ is any $M$-potent ideal of $S$. Then $\left(A \cup N^{\prime} \cup M\right) /\left(N^{\prime} \cup M\right)$ is a nilpotent ideal of $S /\left(N^{\prime} \cup M\right)$. Now by Lemma 2.1 every non-zero f-class of $S /\left(N^{\prime} \cup M\right)$ is an f-class of $S$ in $S-\left(N^{\prime} \cup M\right)$ and is thus an f-class of $S / N^{\prime}$. But by Theorem 3.1, $S / N^{\prime}$ is semisimple; hence $S /\left(N^{\prime} \cup M\right)$ is semisimple, and so has no nilpotent ideal other than the zero ideal. Thus $A \subseteq N^{\prime} \cup M$, and so $N_{M} \subseteq N^{\prime} \cup M$.

In particular, if $S$ has a kernel $K$, then $K \subseteq N^{\prime}$ and so $N_{K} \subseteq N^{\prime}$. It is an easy matter to construct semigroups in which $N_{K} \neq N^{\prime}$. The $K$-radical (previously called the lower radical of $S$ by the author ([7], § 2) ) has been discussed by Schwarz [10] and by Clifford [4].

Following Clifford, we shall say that a semigroup $S$ with zero 0 has "no nilpotent ideals" if it has no nilpotent left, right or two-sided ideals apart from (0). 'If $M$ is an ideal of a semigroup $S$, and if $N_{M}$ is $M$-potent, then $S / N_{M}$ has no nilpotent ideals ([4], Theorem 5.I, with $M$ replacing $K$ ).

THEOREM 3.4. Let $S$ be a semigroup with zero and without nilpotent ideals. Then if $S$ satisfies any one of the conditions $\mathscr{H}_{l}, \mathscr{H}_{r}, \mathscr{H}_{f}$, any non-zero ideal of $S$ contains a non-zero simple ideal of $S$.

Proof. Let $M$ be an ideal of $S$ other than the zero ideal. First suppose that $S$ satisfies $\mathscr{H}_{f}$. From the set of all principal ideals of $S$ generated by non-zero elements of $M$ select a minimal member. This is a minimal ideal of $S$, and since it is non-nilpotent, it is simple.

Next suppose that $S$ satisfies $\mathscr{M}_{l}$. From the set of all principal left ideals of $S$ generated by non-zero elements of $M$ select a minimal member. This is a minimal left ideal of $S$, and so, since $S$ has no nilpotent ideals, it is contained in a minimal two-sided ideal $A$ of $S$ ([4], Lemma 4.2). Evidently $A \subseteq M$, and since $A$ is non-nilpotent it is simple. A similar argument holds when $S$ satisfies $\mathscr{H}_{\text {r }}$.

This result shows that the zero element is, so to speak, ringed with simple $f$-classes.

We conclude with two corollaries which closely resemble results obtained by Schwarz and Clifford ([10], Theorem 42 and [4], Corollary 5.3 respectively), but which proceed from weaker hypotheses.

Corollary 3.5. Let $S$ be a semigroup in which every element has finite order. In addition, let $S$ satisfy the maximal condition on two-sided ideals, and any one of the conditions $\mathscr{M}_{l}, \mathscr{M}_{r}$, $\mathscr{H}_{f}$. Let $M$ be any ideal of $S$. Then $S$ is $M$-potent if and only if it contains no idempotent outside $M$.

Proof. Since $S$ satisfies the maximal condition on two-sided ideals, $N_{M}$ is $M$-potent. Now suppose that $N_{M} \neq S$; then $S / N_{M}$ has no nilpotent ideals. Hence by the theorem, $S / N_{M}$ contains a non-zero simple f-class, $F$ say, and this is completely simple since every 
element of the corresponding principal factor has finite order. Thus $F$ contains an idempotent, and so $S-M$ contains an idempotent. The converse is trivial.

Similarly, we have

CoRollary 3.6. Let $S$ be a semigroup satisfying $\mathscr{H}_{l}, \mathscr{M}_{r}$ and the maximal condition on two-sided ideals. Let $M$ be any ideal of $S$. Then $S$ is $M$-potent if and only if it contains no idempotent outside $M$.

Again $N_{M}$ is $M$-potent. If $N_{M} \neq S$, then $S / N_{M}$ contains a non-zero simple f-class $F$. As in the proof of Theorem 2.3, the two minimal properties ensure that $F$ is completely simple, and so it contains an idempotent; thus $S-M$ contains an idempotent.

In both cases the semigroup $S$ has a kernel $K$, say, by Theorem 1.1, and we may take $M=K$.

\section{REFERENCES}

1. R. Baer and F. Levi, Vollständige irreduzibele Systeme von Gruppenaxiomen, S.-B. Heidelberger Akad. Wiss., Beiträge zur Algebra, 18 (1932), 1-12.

2. A. H. Clifford, Semigroups admitting relative inverses, Ann. of Math. (2), 42 (1941), 10371049.

3. A. H. Clifford, Semigroups containing minimal ideals, Amer. J. Math., 70 (1948), 521-526.

4. A. H. Clifford, Semigroups without nilpotent ideals, Amer. J. Math., 71 (1949), 834-844.

5. R. Croisot, Demi-groupes inversifs et demi-groupes réunions de demi-groupes simples, $A n n$. Sci. École Norm. Sup. (3), 70 (1953), 361-379.

6. J. A. Green, On the structure of semigroups, Ann. of Math. (2), 54 (1951), 163-172.

7. W. D. Munn, On semigroup algebras, Proc. Cambridge Phil. Soc., 51 (1955), 1-15.

8. D. Rees, On semi-groups, Proc. Cambridge Phil. Soc., 36 (1940), 387-400.

9. D. Rees, Note on semi-groups, Proc. Cambridge Phil. Soc., 37 (1941), 434-435.

10. S. Schwarz, Zur Theorie der Halbgruppen, Sbornik Prác. Prirodovedeckej Faculty Slovenskej Univerzity $v$ Bratislave, 6 (1943), 64 pp. [Math. Reviews, 10 (1949), 12.]

11. S. Sehwarz, On the structure of simple semigroups without zero, Czechoslovak. Math.J., 1 (76) (1951), 41-53. [Math. Reviews, 14 (1953), 12.]

The UnIVersity

GLASGOW 